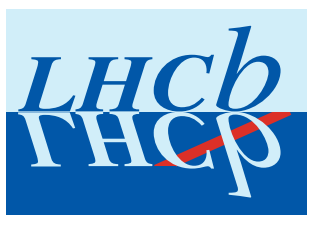

LHCb-CONF-2009-052

VELO

\title{
RESULTS FROM THE \\ FIRST BEAM-INDUCED RECONSTRUCTED TRACKS IN THE LHCB VERTEX LOCATOR *
}

\author{
E. Rodrigues \\ University of Glasgow, Glasgow G12 8QQ, Scotland \\ On behalf of the LHCb VELO Group
}

December 2009

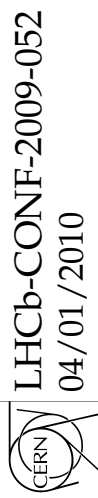

\author{
* Proceedings of the $11^{\text {th }}$ ICATPP Conference on \\ Astroparticle, Particle, Space Physics, Detectors and Medical Physics Applications \\ Villa Olmo, Como (Italy), 5-9 October 2009
}




\begin{abstract}
$\mathrm{LHCb}$ is a dedicated experiment at the LHC to study CP violation and rare $b$ decays. The vertex locator (VELO) is a silicon strip detector designed to measure precisely the production and decay vertices of $B$-mesons. The detector is positioned at $8 \mathrm{~mm}$ of the LHC beams and will operate in an extremely harsh radiation environment. The VELO consists of two retractable detector halves with 21 silicon micro-strip tracking modules each. A module is composed of two $\mathrm{n}^{+}$-on-n $300 \mu \mathrm{m}$ thick half disc sensors with $R$ and $\Phi$ micro-strip geometry. The detectors are operated in vacuum and a bi-phase $\mathrm{CO}_{2}$ cooling system is used. The full system has been operated since June 2008 and its commissioning experience will be reported. During the LHC synchronization tests in August and September 2008, and June 2009 the LHCb detectors measured secondary particles produced by the interaction of the LHC primary beam on a beam dump. About 50,000 tracks were reconstructed in the VELO and they were used to derive the relative timing alignment between the sensors and for the first evaluation of the spatial alignment. Using this track sample the VELO has been aligned to an accuracy of $5 \mu \mathrm{m}$. A single hit resolution of $10 \mu \mathrm{m}$ was obtained at the smallest pitch for tracks of perpendicular incidence. The design and the main components of the detector system are introduced. The commissioning of the detector is reported and the talk will focus on the results obtained using the first beam-induced reconstructed tracks.
\end{abstract}




\section{Introduction}

The LHCb experiment [1] at CERN's Large Hadron Collider (LHC) is dedicated to the study of $\mathrm{CP}$ violation and rare $b$ - and $c$-flavoured hadron decays. The physics goals of $\mathrm{LHCb}$ demand in particular excellent tracking and identification of secondary vertices from long lived particles.

The vertex locator (VELO) [2] is a silicon strip detector designed to precisely measure the production and decay vertices of long-lived mesons, thereby separating primary from secondary vertices. The VELO consists of a row of 21 silicon micro-strip tracking modules, for a total of 84 silicon sensors. A module is composed of two $\mathrm{n}^{+}$-on-n $300 \mu \mathrm{m}$ thick half disc sensors with radial $(R)$ and azimuthal $(\Phi)$ micro-strip geometry. The detector is positioned at $8 \mathrm{~mm}$ of the LHC beams and will operate in an extremely harsh radiation environment. It is separated from the primary LHC vacuum by a thin $(300 \mu \mathrm{m})$ foil and operated in a secondary vacuum with a bi-phase $\mathrm{CO}_{2}$ cooling system. The two retractable detector halves will be opened by $29 \mathrm{~mm}$ during beam injection.

The full system has been operated since June 2008 and its commissioning experience will be outlined in Sec. 2. Section 3 will focus on the VELO achievements obtained with the data samples from the various beam synchronization tests carried out since August 2008.

\section{VELO Commissioning}

Commissioning of the VELO started soon after its installation in 2007. Among the various milestones achieved since then, it is worth mentioning the satisfactory comparisons of noise level with data taken during the modules assembly, the single module operations under neon atmosphere, the subsequent multi-module testing, the full detector half power on for the first time, the first operation in vacuum on the $18^{\text {th }}$ June 2008 followed shortly by the full detector operation under vacuum, cooling down of the modules to their operating temperature of $-5^{\circ} \mathrm{C}$, the successful high-rate tests (at the nominal $1 \mathrm{MHz}$ rate), and the operation under final conditions.

The geometry of the VELO making cosmic tracks unexploitable to commission the detector with real data, an alternative was found in the so-called beam synchronization tests carried out on various occasions as part of the LHC machine commissioning since August 2008. These tests have been crucial in providing data samples of tracks traversing the silicon. The experimental conditions and main results are detailed in the section below. 


\section{Beam Synchronization Tests}

The commissioning of the LHC comprises beam synchronization tests during which, as part of the tests programme, a single bunch of protons is directed along a transfer line between the SPS (pre-)accelerator and the LHC, and dumped onto a beam absorber before entering the LHC tunnel. The beam dump, the TED (Transfer line External beam Dump), is situated about $340 \mathrm{~m}$ from the LHCb detector. Secondary (and tertiary, etc.) tracks travelling at small angles were detected and reconstructed by the VELO.

Several beam synchronization tests were carried out in August and September 2008, and June 2009. The next run is scheduled to take place before the LHC start-up in November; it is foreseen for mid October. The duration of these tests was often extended, upon request of $\mathrm{LHCb}$, as they provided most useful data for the commissioning of the VELO. They typically lasted for two days, with shots being sent every 48 seconds, each of which comprising roughly $5 \cdot 10^{9}$ protons.

A variety of aspects of the VELO commissioning were tested during these special real data runs; notably, the data acquision (DAQ) "recipes" that dictate the acquisition settings, the tuning of the timing settings, the commissioning of the online and offline monitoring tools, the performance of the VELO pattern recognition algorithms, the performance of the alignment procedure and algorithms.

\subsection{Tests in 2008}

The performance of the VELO detector was first assessed from real data with the 2008 synchronization tests, using a system configured with DAQ and timing settings tuned according to calculated cable lengths and known delays. The data quality studies based on the 2200 reconstructed tracks showed reduced performance - e.g. lower cluster finding efficiencies and higher than expected number of noise clusters - because of non-optimal VELO processing parameters and timing settings. The VELO raw signal processing comprises several stages and algorithms for pedestal correction in all sensor channels, common-mode suppression, clusterization thresholds, etc. Subsequent re-processing with tuned parameters showed better reconstruction performance, as expected.

The hit resolution was found to be equivalent to the binary resolution, and consistent with expectations given the average cluster size. The relative VELO module alignment was also determined using this data sample. The modules were found to be displaced relative to the survey positions by less than $10 \mu \mathrm{m}$; the precision of the software alignment being around $5 \mu \mathrm{m}$ for $x$ and $y$ translations and $200 \mu \mathrm{rad}$ for translations around the $z$-axis. 


\subsection{Test in June 2009}

The synchronization tests on the $5^{\text {th }}-6^{\text {th }}$ June 2009 increased the tracks data sample by more than an order of magnitude, bringing it to a total of around 50,000 tracks. All sensors were biased, and the VELO experienced a very stable operation, the cooling keeping the silicon at around $25^{\circ} \mathrm{C}$.

The timing settings were first tuned, sampling in 25 ns intervals around the "nominal" settings for signal in 5 consecutive time slots. The system was then scanned in 4 steps of 6.5 ns intervals, providing 20 time points. A precision of better than 1 ns was achieved.

The ADC spectra (signal shape) were found to precisely match a Landau distribution. The signal-to-noise ratio was found to be $18(20)$ for $R(\Phi)$ sensors.

The pattern recognition pseudo-efficiency was calculated by interpolation, between the first and last hits on the reconstructed tracks; it takes into account detector material inefficiencies, reconstruction and track finding performance. It was found to be around $97 \%$ for $R$ and $\Phi$ sensors. Having the detector halves almost in the closed position, and exploiting the large track sample, allowed for the first time the determination of the distance between the VELO halves using traversing tracks, i.e. tracks with hits in both halves. After moving the VELO halves by $450 \mu \mathrm{m}$ (in $x$ ) the software alignment very successfully determined the applied relative displacement to be $445 \pm 10 \mu \mathrm{m}$.

Figure 1 shows typical residual distributions (versus the sensors strip pitch) for $R$ and $\Phi$ sensors, obtained with this June 2009 data sample of very small angle tracks (i.e. tracks almost parallel to the beamline) with about $90 \%$ of its clusters being composed of a single hit strip. Unsuprisingly, the resolution was found to be marginally better than the binary

resolution. However, one expects a much better resolution from collision data, where tracks will traverse the sensitive silicon strips at larger angles, giving rise to an improved charge sharing between the adjacent strips of multi-strip clusters.

\section{Conclusions}

The vertex locator of LHCb has been fully installed and tested. First-ever beam-induced tracks were reconstructed in August 2008 and the large data sample collected during subsequent similar LHC synchronization tests has provided a wealth of results, proving that the detector performance has achieved its requirements for the experiment's physics goals. $\mathrm{LHCb}$ is ready to start taking collision data by the end of this year when the LHC will provide first collisions. 
R Sensor Number 35

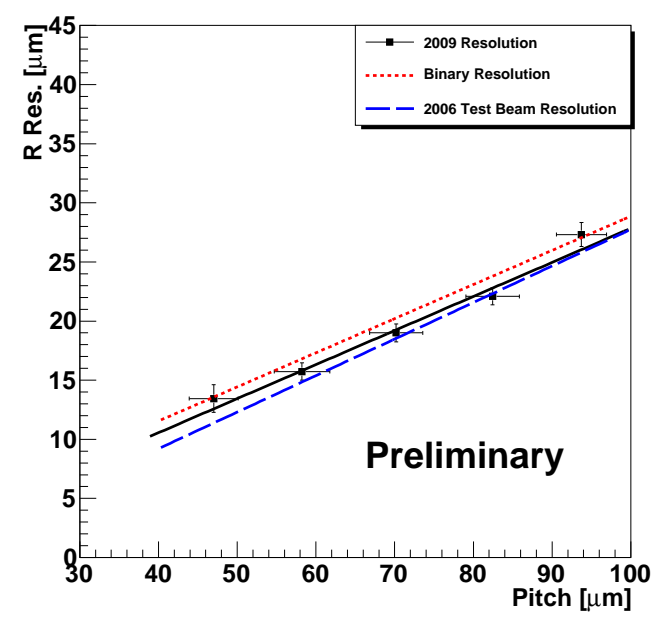

Sensor Number 101

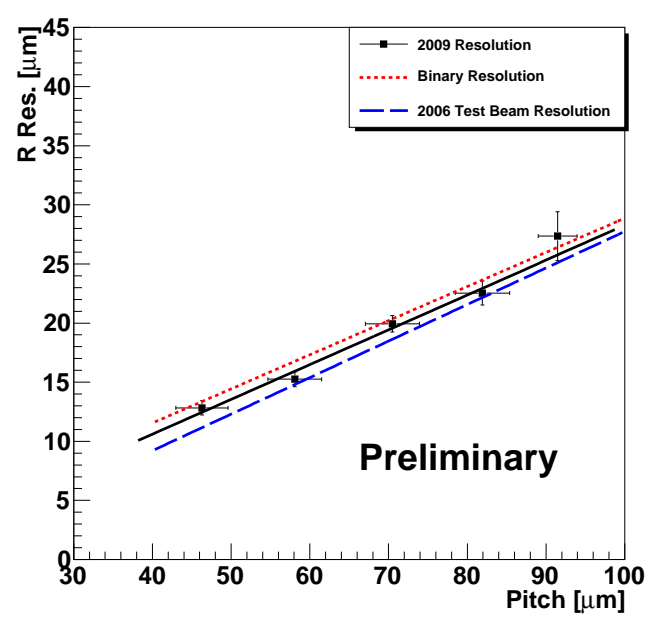

Figure 1: Example of resolution curves for $R$ and $\Phi$ sensors. 


\section{References}

[1] The LHCb Collaboration, The LHCb Detector at the LHC, JINST 3, S08005 (2008).

[2] The LHCb Collaboration, LHCb VELO Technical Design Report, CERN-LHCC-2001011 ; LHCb-TDR-5. 